\title{
Altered gene expression and possible immunodeficiency in cases of sudden infant death syndrome
}

\author{
Linda Ferrante', Torleiv O. Rognum ', Åshild Vege', Ståle Nygård² and Siri H. Opdal ${ }^{1,3}$
}

BACKGROUND: A large number of studies have tried to uncover a genetic predisposition for sudden infant death syndrome (SIDS), but there is still uncertainty concerning the pathogenesis of these deaths. The purpose of this study was to investigate mRNA gene expression in SIDS cases and controls, in order to uncover genes that are differentially expressed in the two groups.

METHODS: Tissue from brain, heart, and liver from 15 SIDS cases and 15 controls were included in the study, and mRNA expression was determined using the Illumina whole genome gene expression DASL HT assay.

RESULTS: Seventeen genes showed significantly altered expression compared to controls, after correction for multiple testing. Three genes involved in the immune system were of particular interest, including the downregulation of MyD88 in tissue from SIDS brains, as well as the downregulation of the genes encoding CCL3 and UNC13 in the liver.

CONCLUSION: These findings indicate that there is an altered expression of genes involved in the inflammatory process in a proportion of SIDS cases, which further strengthen the hypothesis that impaired immune response play a role in this syndrome.

$S_{\text {den }}^{u}$ udden infant death syndrome (SIDS) is defined as the sudden unexpected death of an infant less than one year of age, with onset of the fatal episode apparently occurring during sleep, which remains unexplained after a thorough investigation including performance of complete autopsy, review of the circumstances of death and the clinical history (1). Although the rate has declined dramatically since the "back to sleep campaign", as much as 17 infants succumbed from SIDS in Norway in 2012. This corresponds to a rate of 0,028 per 1,000 live births in 2012.

The decline in SIDS rate is due to new recommendations for infant care, based on knowledge about environmental risk factors. However, a large number of studies have indicated that there might be predisposing genetic factors for SIDS, and so far genes involved in the regulation of the immune system, cardiac function, the serotonergic network and brain function and development have emerged as the most promising $(2,3)$. Despite these observations, the molecular pathogenesis of SIDS remains unclear, and genetic variants with strong dominance in SIDS have not yet been uncovered. The reason might be the lack of homogeneity in the SIDS population, causing a possible gene variant with strong dominance to be hidden. Furthermore, genetic variations may either be the cause of death, or act as a predisposing factor (2).

Thus, an alternative approach is needed, and recently new and more efficient technologies have provided tools that can give extensive information about the genetic diversity of SIDS. A study of messenger RNA (mRNA) expression may thus show the concurrent gene transcription, and thereby bridge the gap between fixed genomic information and dynamic phenotype. By comparing levels of mRNA expression in SIDS and controls, it may be possible to identify disease-associated genes.

The purpose of this study was to investigate gene expression in SIDS cases and controls, in order to uncover genes that are expressed differently between the two groups, and thereby enable us to identify target genes that may contribute to death.

\section{RESULTS}

The Illumina mRNA expression analysis gave satisfactory results in brain tissue from 10 SIDS cases, in heart tissue from 12 SIDS cases, and in liver tissue from 10 cases. The same held true for 10 controls in each of the three organs (Tables 1 and 2). The results of the whole genome gene expression revealed 14 downregulated, and two upregulated genes, for which the false discovery rate (FDR) was below $20 \%$ (Table 3 ). In addition, we included MyD88 which was borderline significantly downregulated in the brain $\left.\left(P=2.7 \times 10^{-5}\right), \mathrm{FDR}=0.38\right)$, as this gene is of particular biological interest. The organ with largest alteration in gene expression was the liver. In this organ, 13 genes were found downregulated, while one gene was upregulated. Furthermore, one gene was upregulated and one downregulated in the heart, while one gene was down regulated in the brain (Table 3).

\footnotetext{
'Department of Pediatric Forensic Medicine, Norwegian Institute of Public Health, Oslo, Norway; ${ }^{2}$ Bioinformatics Core Facility, Institute for Medical Informatics, Oslo University Hospital, Oslo, Norway; ${ }^{3}$ Department of Pathology, Oslo University Hospital, Oslo, Norway. Correspondence: Linda Ferrante (linda.ferrante@fhi.no)

Received 6 October 2015; accepted 23 December 2015; advance online publication 6 April 2016. doi:10.1038/pr.2016.45
} 
Articles $\mid$ Ferranteetal.

Table 1. SIDS cases with microbiology findings

\begin{tabular}{|c|c|c|c|c|c|c|}
\hline Code & Microbiology & Gender & Age (weeks) & Brain & Heart & Liver \\
\hline SIDS1 & Streptococcus pneumonia (blood) & Boy & 36 & & $x$ & $x$ \\
\hline SIDS2 & Negative & Boy & 36 & $x$ & $\mathrm{x}$ & $x$ \\
\hline SIDS4 & Negative & Boy & 12 & $\mathrm{x}$ & $\mathrm{x}$ & \\
\hline SIDS5 & Negative & Boy & 80 & & $x$ & $x$ \\
\hline SIDS8 & Negative & Girl & 4 & $\mathrm{x}$ & $\mathrm{x}$ & $x$ \\
\hline SIDS9 & Staphylococcus aureus (blood) & Girl & 44 & & $x$ & \\
\hline SIDS10 & Negative & Boy & 28 & $x$ & & $x$ \\
\hline SIDS11 & Staphylococcus aureus (lung tissue) & Boy & 28 & $x$ & & \\
\hline SIDS 14 & $\begin{array}{l}\text { Streptococcus pneumonia (nasopharyngeal } \\
\text { secretion and lungs tissue) }\end{array}$ & & & & & \\
\hline SIDS15 & Negative & Boy & 3 & $x$ & $X$ & $x$ \\
\hline
\end{tabular}

The organs included in the Illumina array from each infant are indicated with an $x$. The empty spaces indicate the tissues in which RNA integrity number was considered too low and not included in the assay. Four of the SIDS cases were non-Caucasians, while the rest of the cases included were Caucasians.

SIDS, sudden infant death syndrome.

Table 2. Control cases with microbiology findings

\begin{tabular}{|c|c|c|c|c|c|c|c|}
\hline Code & Microbiology & Ethnicity & Gender & Age (weeks) & Brain & Heart & Liver \\
\hline $\mathrm{C} 1$ & Negative & Caucasian & Boy & 0,5 & & & $\mathrm{x}$ \\
\hline $\mathrm{C} 2$ & $\begin{array}{l}\text { Staphylococcus aureus (tracheal } \\
\text { secretion and lung tissue) }\end{array}$ & Non-Caucasian & Boy & 0,5 & $x$ & & \\
\hline $\mathrm{C} 3$ & Negative & Non-Caucasian & Boy & 144 & $x$ & $x$ & $x$ \\
\hline $\mathrm{C} 4$ & Negative & Non-Caucasian & Boy & 0,1 & & $x$ & $x$ \\
\hline$C 7$ & Negative & Caucasian & Girl & 52 & $x$ & $x$ & $x$ \\
\hline $\mathrm{C} 8$ & Negative & Caucasian & Girl & 0 & $x$ & $x$ & $x$ \\
\hline $\mathrm{C} 9$ & Negative & Caucasian & Girl & 4 & $x$ & & \\
\hline $\mathrm{C} 10$ & Negative & Caucasian & Girl & 80 & & $x$ & $x$ \\
\hline C13 & Negative & Non-Caucasian & Boy & 0 & $x$ & $\mathrm{x}$ & \\
\hline C14 & Negative & Caucasian & Girl & 0 & $\mathrm{x}$ & $\mathrm{x}$ & $x$ \\
\hline $\mathrm{C} 15$ & Negative & Caucasian & Boy & 24 & & $x$ & $x$ \\
\hline
\end{tabular}

The organs included in the Illumina array from each infant are indicated with an $x$. The empty spaces indicate the tissues in which RNA integrity number was considered too low and not included in the assay.

Ethnicity $(P>0.2)$ and age $(P>0.06)$ did not influence the expression of any of the genes in Table 3. The MannWhitney $U$-test for ethnicity and age were calculated against the expression level of every individual using GraphPad prism software version 5.04 for windows, (San Diego, CA).
The ethnicity was clustered to Caucasian and non-Caucasians since a more detailed ethnic origin was difficult to obtain. However, to our knowledge, there is not any known altered gene regulation between different ethnical groups. We choose to calculate this to rule out any possible limitation 
Table 3. Survey of genes with an altered expression in sudden infant death syndrome compared to controls

\begin{tabular}{|c|c|c|c|}
\hline Gene & Organ & Expression & $P$ value \\
\hline $\begin{array}{l}\text { Myeloid differentiation primary } \\
\text { response gene } 88 \text { (MyD88) }\end{array}$ & Brain & Downregulated & $2.7 \times 10^{-5}$ \\
\hline $\begin{array}{l}\text { Chemokine (C-C Motif) Ligand } \\
3 \text { (CCL3) }\end{array}$ & Liver & Downregulated & $6.7 \times 10^{-07}$ \\
\hline F-box protein 24(FBXO24) & Liver & Downregulated & $5.7 \times 10^{-07}$ \\
\hline $\begin{array}{l}\text { Protein Unc-13 Homolog } \\
\text { D(UNC13D) }\end{array}$ & Liver & Downregulated & $2.0 \times 10^{-06}$ \\
\hline Cut-like homeobox 1(CUX1) & Liver & Downregulated & $1.6 \times 10^{-05}$ \\
\hline Zinc finger protein 236 (ZNF236) & Liver & Downregulated & $2.8 \times 10^{-05}$ \\
\hline $\begin{array}{l}\text { SAPS domain family, member } 2 \\
\text { (SAPS2) }\end{array}$ & Liver & Downregulated & $3.1 \times 10^{-05}$ \\
\hline $\begin{array}{l}\text { Intercellular adhesion molecule } \\
4 \text { (ICAM4) }\end{array}$ & Liver & Downregulated & $5.2 \times 10^{-05}$ \\
\hline $\begin{array}{l}\text { Chromosome } 16 \text { open reading } \\
\text { frame } 74 \text { (C16orf74), }\end{array}$ & Liver & Downregulated & $9.2 \times 10^{-05}$ \\
\hline $\begin{array}{l}\text { FBJ murine osteosarcoma viral } \\
\text { oncogene homolog (FOS) }\end{array}$ & Liver & Downregulated & $1.4 \times 10^{-04}$ \\
\hline Kruppel-like factor 4 (KLF4), & Liver & Downregulated & $1.4 \times 10^{-04}$ \\
\hline $\begin{array}{l}\text { NMDA receptor regulated } 2 \\
\text { (NARG2) }\end{array}$ & Liver & Downregulated & $1.4 \times 10^{-04}$ \\
\hline S100A13 & Liver & Downregulated & $1.8 \times 10^{-04}$ \\
\hline Tetraspanin 32 (TSPAN32) & Liver & Downregulated & $1.8 \times 10^{-04}$ \\
\hline Zinc finger protein 167 (ZNF167) & Liver & Upregulated & $1.9 \times 10^{-06}$ \\
\hline $\begin{array}{l}\text { Potassium channel modulatory } \\
\text { factor } 1 \text { (KCMF1) }\end{array}$ & Heart & Upregulated & $1.1 \times 10^{-06}$ \\
\hline Astrotactin 1 (ASTN1) & Heart & Downregulated & $3.0 \times 10^{-06}$ \\
\hline
\end{tabular}

due to ethnic variation patterns at DNA level. There were no significant differences in the expression level between male and female subjects (data not shown). Furthermore, there were no correlation between postmortem time and expression level (data not shown).

Amongst the 17 genes presented in Table 3, the most interesting for this study were those encoding immune related proteins, including CCL3, UNC13D, and MyD88.

\section{MyD88}

MyD88 is an important adapter protein in the signaling pathway of the toll-like receptors which are important components in the immune system. The immune gene MyD88 was found downregulated in brain in SIDS $\left(P=2.7 \times 10^{-5}\right)$, even though not significant after multiple testing correction $(q$-value $=0.38)$ (Figure 1). However, MyD88 was confirmed downregulated by quantitative Real-time polymerase chain reaction (qRT-PCR) $(P=0.01)$. Most of the SIDS cases had very low MyD88 expression compared to the controls. In the SIDS population two cases were reported to have growth of Staphylococcus aureus in lung tissue (SIDS3 and SIDS11) and one case was reported to have growth of both S. aureus and Streptococcus pneumoniae in nasopharyngeal secretion and lung tissue (SIDS14) (Table 1). Also two of the controls had growth of bacteria, one case of

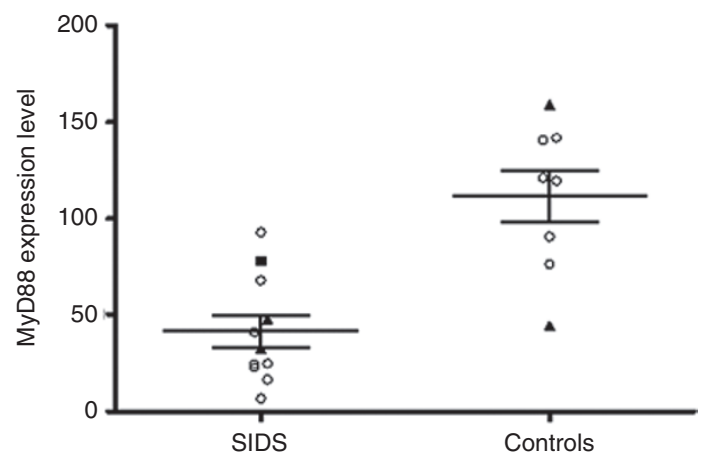

Figure 1. Scatter plot comparing expression of MYD88 in SIDS cases with controls. The triangle represents cases in which S. aureus was detected; the square represents a case in which both $S$. aureus and $S$. pneummoniae were detected.

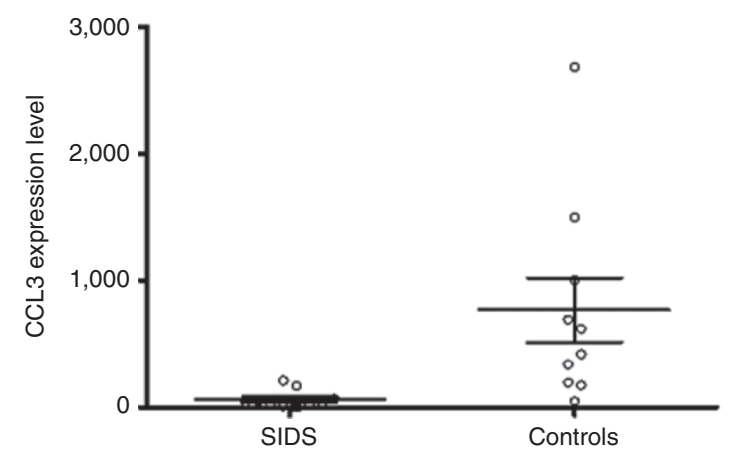

Figure 2. Scatter plot comparing expression of CCL3 in SIDS cases with controls. The triangle represents the case in which S. pneumoniae was detected; the square represents cases in which both $S$. aureus and $S$. pneummoniae were detected.

S. aureus (C2) and one case of S. pneumoniae (C12). The only case with growth of two different bacteria showed high MyD88 expression (SIDS14) (Figure 1; Table 1).

\section{CCL3}

Also the immune gene CCL3 was found to be significantly downregulated in liver in SIDS $\left(P=6.7 \times 10^{-07}\right)$ (Figure 2). One of the cases was found to have growth of both $S$. pneumoniae and $S$. aureus in nasopharyngeal secretion and lungs (SIDS14), while one case was found to have growth of S. pneumoniae (SIDS1) in blood. Both cases showed particularly low CCL3 expression level (Figure 2). None of the controls with expression data in CCL3 had growth of bacteria.

\section{UNC13D}

UNC13D was found significantly down regulated in liver samples in the SIDS cases $\left(P=2.0 \times 10^{-6}\right)$ (Figure 3). One of the cases was found to have growth of both $S$. pneumoniae and $S$. aureus in nasopharyngeal secretion and lungs (SIDS14) while one case was found to have growth of S. pneumoniae (SIDS1) in blood. Both cases were found to have downregulated UNC13D. Although the case with only $S$. pneumoniae present showed a little higher expression pattern, it still showed lower activity than the controls (Figure 3). 


\section{Articles $\mid$ Ferrante et al.}

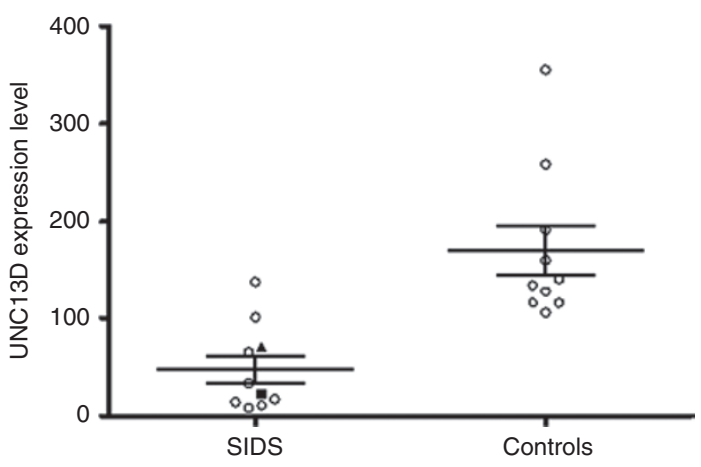

Figure 3. Scatter plot comparing expression of UNC13D in SIDS cases with controls. The triangle represents the case in which S. pneumoniae was detected; the square represents cases in which both S. aureus and $S$. pneumoniae were detected.

\section{DISCUSSION}

The main findings of this study are the observed downregulation of 15 genes, as well as an upregulation of two genes, in SIDS cases compared to controls (Table 3 ).

In our opinion, the downregulation of MyD88 in brain tissue from SIDS victims $\left(P=2.7 \times 10^{-5}\right)$ is the most interesting finding, even though the gene was not statistically significant after correction for multiple testing $(q=0.35)$. However, the downregulation of this gene was verified using qPCR. MyD88 is a cytosolic adapter protein that plays a central role in the innate and adaptive immune response, and is an important signal transducer in pathways that regulate the activation of several proinflammatory genes. This makes MyD88 important for Toll-like receptor (TLR) pathways and for proinflammatory gene expression. There are several studies indicating an immune-activation in cases of SIDS (4-7). In spite of absence of fatal inflammatory reaction, a significant proportion of SIDS victims have levels of IL- 6 in the cerebrospinal fluid in the same range as infants with fatal infection $(5,8)$. Furthermore, SIDS cases have significantly more frequent Helicobacter pylori in their stools than controls (9), and the prevalence of $S$. aureus in nasopharyngeal flora is significantly higher in SIDS cases compared to age-matched controls (4). These observations are interesting as Von Bernuth et al. (10), have reported nine infants and children with autosomal recessive MyD88 deficiency that suffered life-threatening often recurrent bacterial infection. Three of the nine children in their study died between 1 and 11 mo of age.

The findings reporting immune activation in SIDS might indicate that some immunological pathways not have an optimal signal transduction. This could result in that the observed immune activation not fights down the infection successfully. A defective MyD88 could compromise one of the TIL-pathways, leaving important immunological pathways less effective.

MyD88 deficiency may be the result of known mutations in the MyD88 gene. It is known that the stop mutation in exon 1 (E66X), the deletion E52del, and the missense mutation L93P all give low levels of Myd88, while the R196C interferes with the ability to associate with IL-1R (10,11). Myd88 deficiency generally abolishes cytokine responses after TLR activation, and may therefore supress an optimal immune reaction. The mutations have been found to predispose to recurrent lifethreatening pyogenic bacterial infections, and patients with these mutations are particularly vulnerable to Pseudomonas aeruginosa, S. pneumonia, and S. aureus, especially in infancy and early childhood (12).

Whether the gene expression divergence of MyD88 observed between SIDS and the controls in this study is caused by any of these mutations, by de novo mutations in the MyD88 gene or is caused by a completely other biological processes is not known. Whether the reduced expression level of MyD88 seen in SIDS is caused by mutations in the MyD88 gene may be confirmed by sequencing the gene. Exome sequencing studies are being planned for the MyD88 gene, genes in the TLR pathway, and the other genes reported in the study as this is essential for a better understanding on our current findings. It is also interesting that two of the cases included in the present study were reported to have growth of $S$. aureus while one case had growth of both S. aureus and S. pneumoniae. The one control case (C2) with growth of S. aureus, showed a very low Myd88 expression, corresponding to the median of the SIDS group (Figure 1). The death of this control case happened one day after a minor surgery procedure, possibly due to blood loss. There were no signs of immune activation according to microscopic evaluation. A potential depressed immune reaction cannot be excluded as contributing to death in this case.

The observed altered gene expression can be caused by a number of biodynamic processes. Nevertheless, there is a difference in the mRNA expression between the SIDS group and the control group suggesting the SIDS population do have a different pattern of expression for these particular genes. The study therefore provides us with new candidate genes that might be involved as a genetic predisposing factor for SIDS.

The age, and hence the developmental stages of the individuals could also influence the gene expression and at least in part explain the mRNA expression pattern observed in this study. However, with such an approach to the results we would expect the same pattern in both SIDS and controls as the two groups are age matched as best as possible.

One may also speculate that the transcriptional downregulation of MyD88 in the brain of SIDS might be caused by a postinflammation state. It is known that several inflammatory processes in the brain, like stroke, infection, and other diseases, can activate TLR and lead to an upregulation of MyD88 (13). After this activation, a significant downregulation of MyD88-mRNA occurs, probably to limit the extent and duration of the inflammation. In a study of Helicobacter Pylori in humans, postinfection negative regulation of inflammation was achieved by downregulation of MyD88 through the upregulation of the miRNA Let-7b (14). Interestingly, Let-7b is reported to be upregulated in the brains of SIDS infants (15).

The liver plays a major role in carbohydrate, protein, and lipid metabolism, and is also involved in the production of components involved in the immune response. Inborn metabolic disorders, especially in fatty acid metabolism, may be fatal if undetected. A study by Boles et al. (16) revealed that 
about $5 \%$ of the cases initially diagnosed as SIDS actually were due to different fatty acid oxidation defects. However, only two cases had the common A985G mutation in the medium-chain acyl-CoA dehydrogenase gene, emphasizing the importance of investigation also other metabolic disorders in cases of sudden unexpected infant death. One might speculate that the altered gene expression in several genes in the liver found in this study might indicate a larger involvement of the liver in SIDS that previously thought. CCL3, also known as macrophage inflammatory protein $1 \alpha$ (MIP-1 $\alpha)$ is critical for liver NK cell inflammation. CCL3 is a chemokine strongly expressed in several cell types that recruits many different cells to a site of infection. The downregulation of CCL3 in the liver of SIDS victims may therefore be of importance since effective delivery of innate immune mechanisms depends on recruitment of cells to the sites of infection. A study by Takahashi et al. (17) in mice showed that CCL3 is essential for the resistance to sepsis. It is also reported a significant role of CCL3 action for IFN- $\alpha / \beta$ in promoting expression of MIP- $1 \alpha$ and the accumulation of NK cells in liver during murine CMV infection (18).

UNC13D, also known as Munc13-4, is a part of the perforin (PRF1)-dependent cytotoxic pathway. This gene was also found significantly downregulated in liver in SIDS cases. The Munc13-4 protein is thought to contribute to the priming of secretary granules before they fuse in to the plasma cell membrane, and mutations in this gene may impair the delivery of effector proteins (19). Deficiencies in such mechanisms may most likely also interfere with the immune response. Studies have shown mutations in the Munc13-4 gene in children and young adults with haemophagocytic lymphohistocytosis (HLH), which is a collection of severe immune disorders of inherited and acquired forms (20). A study by Rohr et al. (21) has shown an overlap between atypical familial HLH due to mutation in the UNC13 and Syntaxin-Binding Protein 2 (STXBP2) and immunodeficiency diseases. A novel mutation in the UNC13D has furthermore been reported in a Dutch population with HLH, and the authors conclude that this mutation affects the function of the protein (22).

One might speculate that the observed downregulation of CCL3 and UNC13D in liver in the SIDS cases included in our study could be a part of a dysfunction within the immune communication, making the infant vulnerable to infections. One SIDS case in this group was reported with $S$. pneumoniae and one with $S$. pneumoniae and $S$. aureus in both nasopharyngeal secretion and lungs, and at least for these cases one might speculate that the low levels of CCL3 and Munc13-4 might have been of importance.

For the remaining genes in Table 3 with an altered expression in SIDS compared to controls the relevance is more difficult to interpret, as there is little knowledge about most of these genes and the proteins they are encoding. It is however interesting that several of the genes found downregulated in liver in SIDS, including S100A13 and TSPAN32, have functional roles in cellular processes such as cell cycle progression, membrane fusion, proliferation, and adaptive immunity (23). FOS is suggested to be a genetic regulator for cellular mechanisms mediating neuronal excitability and survival (24). ICAM4 is a member of the immunoglobulin (Ig) superfamily and encodes the Landsteiner-Wiener (LW) blood group antigens. It has been speculated that ICAM4 may be involved in regulation of red cell turnover (25). One might speculate that if these genes are of importance with regard to SIDS it is as factors contributing to a suboptimal development of cellular processes important, among other things, for the immune response.

Cardiac disorders, in particular the long QT syndrome, is known to be involved in several cases of sudden unexpected infant death. The most common involved genes in long QT syndrome have been investigated in all cases included in this study, and are exclusion criteria for giving the SIDS diagnosis. All cases included in the study were screened for known LQT mutations. The absence of any heart-specific genes can be partly explained by this screening.

Prone sleeping is a well-known risk factor for SIDS. It is shown that the prone sleeping position results in increased numbers of bacteria, as well as an increase in the variety of species in nasal secretion of infants with respiratory virus infections (26). One might speculate that in infants that are in a vulnerable immunological state, in part due to a decreased level of Myd88, an increased load of infectious agents due to prone sleeping might trigger an unfavorable immunological response.

It is important to emphasize on some of the limitations involved in the study. Using postmortem samples for estimation of gene expression may be challenging, due to RNA degradation. We have tried to limit this by using an optimized autopsy protocol, harvest all samples at the same time as soon as possible after death, and preserve the samples in RNA later, a solution that is developed to rapidly stop any biological activity in the tissue.

We are aware that the number of cases is relatively small, but despite this we believe that the results from this study are of importance. It is however necessary to confirm the findings in larger SIDS populations. However, pure SIDS is a relatively rare condition, and to accumulate a large number of suitable controls is even more difficult. The best controls would be accidental deaths in healthy infants, however such deaths are rare and therefore challenging to obtain.

Another issue may be the age difference between SIDS cases and controls, as the SIDS cases have an average age of $21.3 \mathrm{wk}$ while the controls have a slightly higher average age of $24.7 \mathrm{wk}$ $(P=0.006)$. The tendency to a difference is mainly due to two of the controls being older than the rest of the cases (144 and $80 \mathrm{wk}$, Table 2). When comparing all gene expression with postnatal age, the expression did not seem to change with increasing age. Furthermore, there were no differences in expression level when comparing male and female, indicating that the expression differences seen in this study not is gender specific.

There is difference in ethnicity between the groups, while the SIDS cases were all Caucasians; the control group consisted of seven non-Caucasians and eight Caucasians. When comparing the expression in Caucasians and non-Caucasians, no differences in the expression pattern between these two populations 


\section{Articles $\mid$ Ferrante et al.}

were found $(P>0.2)$. Based on these findings, it seems justified to assume that our observed expression results indicate altered gene expression in SIDS, and not merely results from artefacts caused by the selection of cases.

Gene expression studies have previously been considered to be an unsuitable method for studies on postmortem tissue, due to the nature of the RNA molecules, which are more prone to degradation, and are more unstable than the DNA molecule. Recent studies have explored the RNA stability in different postmortem tissues $(27,28)$. One study looked at stability of RNA molecules in different organs and showed that RNA remained unaffected in brain tissue, cartilage, tendon ligament, and lung tissue for up to $96 \mathrm{~h}$ postmortem (28). It is also shown that RNA from post-mortem tissue harvested up to $24 \mathrm{~h}$ after death can be used to identify differences in biological relevant expression patterns (29). During the past years, the technology has also improved, and special analytical assays for low quality RNA have been developed. We are, therefore, confident that expression studies using postmortem tissue could be successful and provide valuable information for SIDS research. RNA integrity number in the samples included in this study indicates that all tissues analyzed were of adequate quality.

Molecular studies have disclosed several polymorphisms located in immune genes that are associated with SIDS, including genes encoding complement component $\mathrm{C} 4$ and several interleukins (30-35). Considering this immunological vulnerability in SIDS, it is possible that some infants do not have the biological capacity to fight an infection in an effective manner. However, further studies are necessary in order to understand the altered gene expression observed in SIDS in this study. Therefore, to better understand what causes the observed mRNA expression changes, it is imperative a to perform exome sequencing of all the genes reported in this study, together with other genes within the TLR pathway. Such a study is already being designed.

\section{Conclusion}

Overall, these data suggest an altered expression of several genes in SIDS victims compared to controls. Several of these genes are involved in the inflammatory process, which confirms an immunological vulnerability in SIDS. Whether the altered gene expressions observed in this study are due to mutations in the respective genes is yet to be determined.

\section{METHODS}

\section{Subjects}

The subjects investigated were cases of sudden infant death autopsied at the Norwegian Institute of Public Health, in the years 2006-2012. In total, 15 SIDS cases and 15 age-matched controls that suffered explainable death were included in this study (Table 4). The autopsies were performed using a standard protocol, which includes evaluation of the circumstances of death, review of medical and family history, radiographic examination, and a thorough autopsy with extensive histological and microbiological examinations, as well as neuropathological and toxicological examination. The SIDS cases have been classified according to the criteria of the Nordic study of SIDS as pure SIDS (no cause of death revealed) (36). The autopsies were performed within $7-51 \mathrm{~h}$, median postmortem time for SIDS cases was $24.3(7.2-53 \mathrm{~h})$ and for controls $24.0(6.6-65.7 \mathrm{~h})$. In four of the SIDS cases, there was growth of $S$. aureus; however, this was not considered relevant to the cause of death in these cases (Table 1). In the control population, only two cases were reported to have microbiology findings (Table 2).

In one case included in the control group, there was growth of S. aureus; in the other, S. pneumonia growth (Table 2). None of the reported microbiological findings were regarded as causing or contributing to death in any of the controls. The control population was carefully selected, and there was no death within the included cases caused by infection, medium-chain acyl-CoA dehydrogenase deficiency, or heart disease. In the included accidents, there was no trauma to the organs sampled in this study.

The gene expression data is published at Gene Expression Omnibus with series record GSE70422.

\section{Ethics}

The study was reviewed and approved by The National Committees for Research and Ethics in Norway. The Committees have also given the study exemption for obtaining parental consent.

Table 4. SIDS cases and controls

\begin{tabular}{|c|c|c|c|c|}
\hline Group & Number of cases & Cause of death & Gender (M/F) & Age, median (range) \\
\hline SIDS & 15 & & $12 / 3$ & $3 \mathrm{mo}(0.5-20 \mathrm{mo})$ \\
\hline \multirow[t]{8}{*}{ Controls } & 15 & 4 Disease & $8 / 7$ & $0.5 \mathrm{mo}(0-36 \mathrm{mo})$ \\
\hline & & 1 Medium-chain acyl-CoA dehydrogenase deficiency & & \\
\hline & & 1 Heart failure & & \\
\hline & & 5 Labor complications & & \\
\hline & & 4 Accidents & & \\
\hline & & 1 Strangulation & & \\
\hline & & 1 Car accident & & \\
\hline & & 1 Suffocation & & \\
\hline
\end{tabular}




\section{RNA Isolation and Gene Expression}

In this study, we chose the sample tissue from the right temporal lobe of the brain, the right lobe of the liver, and from the apex of the heart. Tissue from the liver was chosen due to its important roles in the metabolism, while the heart were chosen based on the involvement of cardiac disorders in sudden unexpected death. The temporal lobe of the brain was considered to be of interest due to studies on temporal lobe seizure and SIDS (37). Tissue from the brainstem would have been of interest, but was not possible to sample due to its use for diagnostic purposes. The tissue were collected during autopsy, stored in RNA-later, and frozen in $-70{ }^{\circ} \mathrm{C}$ until RNA isolation. Samples were then kept in RNA-later Stabilization Solution (Thermo Fisher Scientific, Waltham, MA) before disrupted using TissueRuptor (Qiagen, Valencia, CA). The RNA was isolated from $\sim 5-44 \mathrm{mg}$ dissected tissue using Qiagen RNeasymini kit (Qiagen) according to manufacturer's protocol. The samples had A260/A280 absorbance ratio $\geq 2.0$. The RNA integrity was evaluated using the RNA 6000 Nano Kit (Agilent Technologies, Santa Clara, CA) in the 2100 Bioanalyzer system (Agilent Technologies). Only samples with a RNA integrity number between $5.6-8.9$ were included (15 SIDS cases and 15 controls).

The isolated RNA samples were further used for whole genome gene expression analysis and $400 \mathrm{ng}$ total RNA was amplified and labeled using Whole-Genome DASL HT kit (Illumina, San Diego, CA), which quantifies 29,378 transcripts. This was done according to the manufacturer's protocol. Beadchips were scanned using the iScan (Illumina) and data were exported using the GenomeStudio software version 11 (Illumina)

The assay is designed to improve the capacity of the target set and retained the ability to profile partially degraded RNA samples. To verify the results from the illumina results, quantitative real-time PCR (qPCR) was performed using the 7900HT Real-Time PCR System (Life Technologies, Darmstadt, Germany). The High Capacity RNAto-cDNA master mix (Life Technologies) was used to synthesize cDNA. The expression levels of MyD88 (assay ID: Hs01573837_g1) and CCL3 (assay ID: Hs04194942_s1) were determined using the TaqMan Gene Expression Assays (Life Technologies) as described by the manufacturer. The relative expression levels were determined using the comparative threshold cycle $\left(2^{-\Delta \Delta \mathrm{Ct}}\right)$ method $(38,39)$, using TBP (assay ID: Hs00427620_m1) as endogenous reference.

\section{Microarray Data Analyses}

The microarray data were preprocessed with the lumiB Bioconductor package (40), where background adjustment was performed with the bgAdjust.affy method. Next, the microarray data were quantile normalized (41), normalizing samples from the same tissue type together. This is based on an observation from a principal component analysis (PCA) plot, showing that type of tissue is more crucial for gene expression than the diagnosis of death (41). For every gene and every group comparison, a two-sampled $t$-test is performed, followed by a correction for multiple testing where Benjamini Hochberg FDR is calculated (42). A FDR of $20 \%$ was used as significance threshold. To exclude influences on the expression pattern due to different ethnic origin and gender, Mann-Whitney $U$-test was performed using GraphPad Prism (San Diego, CA). To disclose possible expression variance in the study population due to age differences and postmortem time, a linear regression analysis was calculated. All microarray data are MIAME compliant and the raw data have been deposited in the NCBI Gene Expression Omnibus under accession number GSE70422.

\section{ACKNOWLEDGMENT}

The study was supported by the Norwegian SIDS and Stillbirth Society. We also would like to thank Ingvar Jon Rognum for valuable statistical assistance.

\section{STATEMENT OF FINANCIAL SUPPORT}

All phases of this study were supported by Norwegian SIDS and Stillbirth Society.

Disclosure: All authors have indicated they have no financial relationships relevant to this article to disclose.

\section{REFERENCES}

1. Krous HF, Beckwith JB, Byard RW, et al. Sudden infant death syndrome and unclassified sudden infant deaths: a definitional and diagnostic approach. Pediatrics 2004;114:234-8.

2. Opdal SH, Rognum TO. Gene variants predisposing to SIDS: current knowledge. Forensic Sci Med Pathol 2011;7:26-36.

3. Ferrante L, Opdal SH. Sudden infant death syndrome and the genetics of inflammation. Front Immunol 2015;6:63.

4. Blackwell CC, MacKenzie DA, James VS, et al. Toxigenic bacteria and sudden infant death syndrome (SIDS): nasopharyngeal flora during the first year of life. FEMS Immunol Med Microbiol 1999;25:51-8.

5. Vege A, Rognum TO, Anestad G. IL-6 cerebrospinal fluid levels are related to laryngeal IgA and epithelial HLA-DR response in sudden infant death syndrome. Pediatr Res 1999;45:803-9.

6. Vege A, Rognum TO, Scott H, Aasen AO, Saugstad OD. SIDS cases have increased levels of interleukin-6 in cerebrospinal fluid. Acta Paediatr 1995;84:193-6.

7. Thrane PS, Rognum TO, Brandtzaeg P. Up-regulated epithelial expression of HLA-DR and secretory component in salivary glands: reflection of mucosal immunostimulation in sudden infant death syndrome. Pediatr Res 1994;35:625-8.

8. Rognum IJ, Haynes RL, Vege A, Yang M, Rognum TO, Kinney HC. Interleukin- 6 and the serotonergic system of the medulla oblongata in the sudden infant death syndrome. Acta Neuropathol 2009;118:519-30.

9. Stray-Pedersen A, Vege A, Rognum TO. Helicobacter pylori antigen in stool is associated with SIDS and sudden infant deaths due to infectious disease. Pediatr Res 2008;64:405-10.

10. von Bernuth H, Picard C, Jin Z, et al. Pyogenic bacterial infections in humans with MyD88 deficiency. Science 2008;321:691-6.

11. Conway DH, Dara J, Bagashev A, Sullivan KE. Myeloid differentiation primary response gene 88 (MyD88) deficiency in a large kindred. J Allergy Clin Immunol 2010;126:172-5.

12. Picard C, von Bernuth $H$, Ghandil $P$, et al. Clinical features and outcome of patients with IRAK-4 and MyD88 deficiency. Medicine (Baltimore) 2010;89:403-25.

13. Li GZ, Zhang Y, Zhao JB, Wu GJ, Su XF, Hang CH. Expression of myeloid differentiation primary response protein 88 (Myd88) in the cerebral cortex after experimental traumatic brain injury in rats. Brain Res 2011;1396: 96-104.

14. Teng GG, Wang WH, Dai Y, Wang SJ, Chu YX, Li J. Let-7b is involved in the inflammation and immune responses associated with Helicobacter pylori infection by targeting Toll-like receptor 4. PLoS One 2013;8:e56709.

15. Courts C, Grabmüller M, Madea B. Dysregulation of heart and brain specific micro-RNA in sudden infant death syndrome. Forensic Sci Int 2013;228:70-4.

16. Boles RG, Buck EA, Blitzer MG, et al. Retrospective biochemical screening of fatty acid oxidation disorders in postmortem livers of 418 cases of sudden death in the first year of life. J Pediatr 1998;132:924-33.

17. Takahashi H, Tashiro T, Miyazaki M, Kobayashi M, Pollard RB, Suzuki F. An essential role of macrophage inflammatory protein 1alpha/CCL3 on the expression of host's innate immunities against infectious complications. J Leukoc Biol 2002;72:1190-7.

18. Salazar-Mather TP, Lewis CA, Biron CA. Type I interferons regulate inflammatory cell trafficking and macrophage inflammatory protein 1alpha delivery to the liver. J Clin Invest 2002;110:321-30.

19. Feldmann J, Callebaut I, Raposo G, et al. Munc13-4 is essential for cytolytic granules fusion and is mutated in a form of familial hemophagocytic lymphohistiocytosis (FHL3). Cell 2003;115:461-73.

20. Santoro A, Cannella S, Bossi G, et al. Novel Munc13-4 mutations in children and young adult patients with haemophagocytic lymphohistiocytosis. J Med Genet 2006;43:953-60.

21. Rohr J, Beutel K, Maul-Pavicic A, et al. Atypical familial hemophagocytic lymphohistiocytosis due to mutations in UNC13D and STXBP2 overlaps with primary immunodeficiency diseases. Haematologica 2010;95: 2080-7.

22. Elstak ED, te Loo M, Tesselaar K, et al. A novel Dutch mutation in UNC13D reveals an essential role of the $\mathrm{C} 2 \mathrm{~B}$ domain in munc13-4 function. Pediatr Blood Cancer 2012;58:598-605. 


\section{Articles | Ferrante et al.}

23. Tarrant JM, Groom J, Metcalf D, et al. The absence of Tssc6, a member of the tetraspanin superfamily, does not affect lymphoid development but enhances in vitro T-cell proliferative responses. Mol Cell Biol 2002;22:5006-18.

24. Zhang J, Zhang D, McQuade JS, Behbehani M, Tsien JZ, Xu M. c-fos regulates neuronal excitability and survival. Nat Genet 2002;30:416-20.

25. Bailly P, Tontti E, Hermand P, Cartron JP, Gahmberg CG. The red cell LW blood group protein is an intercellular adhesion molecule which binds to CD11/CD18 leukocyte integrins. Eur J Immunol 1995;25:3316-20.

26. Harrison LM, Morris JA, Telford DR, Brown SM, Jones K. Sleeping position in infants over 6 months of age: implications for theories of sudden infant death syndrome. FEMS Immunol Med Microbiol 1999;25:29-35.

27. Bahar B, Monahan FJ, Moloney AP, Schmidt O, MacHugh DE, Sweeney T. Long-term stability of RNA in post-mortem bovine skeletal muscle, liver and subcutaneous adipose tissues. BMC Mol Biol 2007;8:108.

28. Marchuk L, Sciore P, Reno C, Frank CB, Hart DA. Postmortem stability of total RNA isolated from rabbit ligament, tendon and cartilage. Biochim Biophys Acta 1998;1379:171-7.

29. Gupta S, Halushka MK, Hilton GM, Arking DE. Postmortem cardiac tissue maintains gene expression profile even after late harvesting. BMC Genomics 2012;13:26.

30. Opdal SH, Vege A, Stave AK, Rognum TO. The complement component C4 in sudden infant death. Eur J Pediatr 1999;158:210-2.

31. Dashash M, Pravica V, Hutchinson IV, Barson AJ, Drucker DB. Association of sudden infant death syndrome with VEGF and IL-6 gene polymorphisms. Hum Immunol 2006;67:627-33.

32. Moscovis SM, Gordon AE, Al Madani OM, et al. IL6 G-174C associated with sudden infant death syndrome in a Caucasian Australian cohort. Hum Immunol 2006;67:819-25.
33. Ferrante L, Opdal SH, Vege A, Rognum TO. TNF-alpha promoter polymorphisms in sudden infant death. Hum Immunol 2008;69:368-73.

34. Ferrante L, Opdal SH, Vege A, Rognum TO. IL-1 gene cluster polymorphisms and sudden infant death syndrome. Hum Immunol 2010;71: 402-6.

35. Ferrante L, Opdal SH, Vege A, Rognum T. Cytokine gene polymorphisms and sudden infant death syndrome. Acta Paediatr 2010;99:384-8.

36. Gregersen M, Rajs J, Laursen H, Baandrup U, Frederiksen P, Gidlund E, et al. Pathologic criteria for the Nordic study of SIDS. In: Rognum TO, editor. Sudden Infant Death Syndrome, New Trends in the Nineties. Oslo: Scandinavian University Press; 1995:50-8.

37. Kinney HC, Cryan JB, Haynes RL, et al. Dentate gyrus abnormalities in sudden unexplained death in infants: morphological marker of underlying brain vulnerability. Acta Neuropathol 2015;129:65-80.

38. Livak KJ, Schmittgen TD. Analysis of relative gene expression data using real-time quantitative PCR and the 2(-Delta Delta C(T)) Method. Methods 2001;25:402-8.

39. Pfaffl MW. A new mathematical model for relative quantification in realtime RT-PCR. Nucleic Acids Res 2001;29:e45.

40. Gentleman RC, Carey VJ, Bates DM, et al. Bioconductor: open software development for computational biology and bioinformatics. Genome Biol 2004;5:R80.

41. Bolstad BM, Irizarry RA, Astrand M, Speed TP. A comparison of normalization methods for high density oligonucleotide array data based on variance and bias. Bioinformatics 2003;19:185-93.

42. Benjamini Y, Hochberg Y. Controlling the false discovery rate: a practical and powerful approach to multiple testing. J Royal Statistical Society Series B. 1995;57:189-300. 